Bolm Inst. oceanogr., S Paulo, 34 (ünico):1-12, 1986

\title{
INCIDÊNCIA DE ISÓPODES PARASITAS (CYMOTHOIDAE) EM PEIXES DA PLATA- FORMA CONTINENTAL BRASILEIRA
}

Silvia Maria Sartor*

Instituto Oceanogrä́fico da Unive: sidade de São Paulo (Caixa Postal 9075, 01051 São Paulo, SP)

\section{Synopsis}

In the present paper 90.418 individuals betonging to 186 species of fish were examined. The samples were taken on the southeasterm Brazilian shelf, from Cabo de São Tomé (RJ) ( $\left.22^{\circ} 27^{\prime} S\right)$ to Torres (RJ) $\left(29^{\circ} 21^{\prime} S\right)$ - Brazil. The present results indicate the presence of 12 different parasitic species of Cymothoidae and 13 host-species of fish. A high degree of specificity between Cymothoa $s p_{1} * *$ and the host, Chloroscombrus chrysurus (Pisces, Carangidae) was disclosed. Although Lironeca redmanni is not specific-parasite, it was found mainly on cynoscion striatus (Pisces, Sciaenidae).

Descriptors: Parasites, Parasitism, Isopoda, Cymothoidae, Marine fishes, Carangidae, Sciaenidae, Continental shelf, Southeastern Brazilian coast, R/V "Prof. W. Besnard".

Descritores: Parasitas, Parasitismo, Isopoda, Cymothoidae, Peixes marinhos, Carangidae, Sciaenidae, Plataforma continental, Costa sudeste: Brasil, N/Oc. "Prof. W. Besnard".

\section{Introdução}

Existem vārias espécies de isōpodes que apresentam comportamento parasitário, sendo que peixes e crustáceos, incluindo os próprios isópodes, são encontrados como hospedeiros (Schultz, 1969). As espécies da família Cymothoidae, parasitas de peixes, têm especial interesse, uma vez que muitos hospedeiros são economicamente importantes (Schultz, op. cit.; Novotny \& Mahnken, 1971; Moreira \& Sadowsky, 1978). Além disto, foi visto também que a incidência de parasitismo pode ser alta em determinadas regiões (Bowman, 1960; Trilles, 1962, 1972; Tuê, 1963; Rouse, 1969).

Trabalhos recentes tentam explicar os motivos que levam o parasita a ocupar determinada posição no corpo do hospedeiro, tal como dentro das cavidades bucal e branquial, e externamente, sobre o corpo do mesmo (Lagarrique \& Trilles, 1969; Morton, 1974).

No Brasil, até o momento, pouco existe sobre o assunto, não havendo sequer

(*) Pös-Graduanda do Departamento de Oceanografia Biolögica.

(**) Cymothoa $\mathrm{sp}_{1}$, foi descrita pelo autor como Cymothoa liannea em trabalho a ser publicado em Crustaceana.

Publ. n. 647 do Inst. oceanogr. da Usp. um levantamento atualizado sobre as espécies parasitas e parasitadas. Apenas 30 espécies de parasitas foram registrados, incluindo exemplares marinhos e fluviais (Dana, 1852; Schioedte \& Meinert, 1884; Castro, 1955 e Szidat \& Schubart, 1960). Ligeiras observações ou comentários sobre cimotoídeos brasileiros são apresentados por Cordero (1937), Carvalho (1939), Castro \& Machado Filho (1946) e Moreira (1972, 1973, 1977).

0 presente trabalho relata a ocorrência de 12 espécies de isópodes parasitas presentes em 13 espécies de peixes e discute a existência de especificidade nos parasitas assinalados.

\section{Material e métodos}

0 material examinado foi obtido durante o projeto FAUNEC (Estudo da Fauna Nectônica), com o N/Oc. Prof. "W. Besnard", nas quatro estações do ano de 1975, na plataforma continental centro-sul do Brasil, entre $22^{\circ} 27^{\prime} 00^{\prime \prime} \mathrm{s}$ (Cabo de São Tomé-RS) e $29^{\circ} 21^{\prime} 00^{\prime \prime S}$ (Torres-RS). As Figuras 1-4 indicam a posição das estações de coleta de peixes parasitados.

Os peixes foram coletados com rede-de-arrasto com portas (tipo "otter-traw1") e congelados a $-10 /-25^{\circ} \mathrm{C}$ nas câmaras frigoríficas do navio. As 


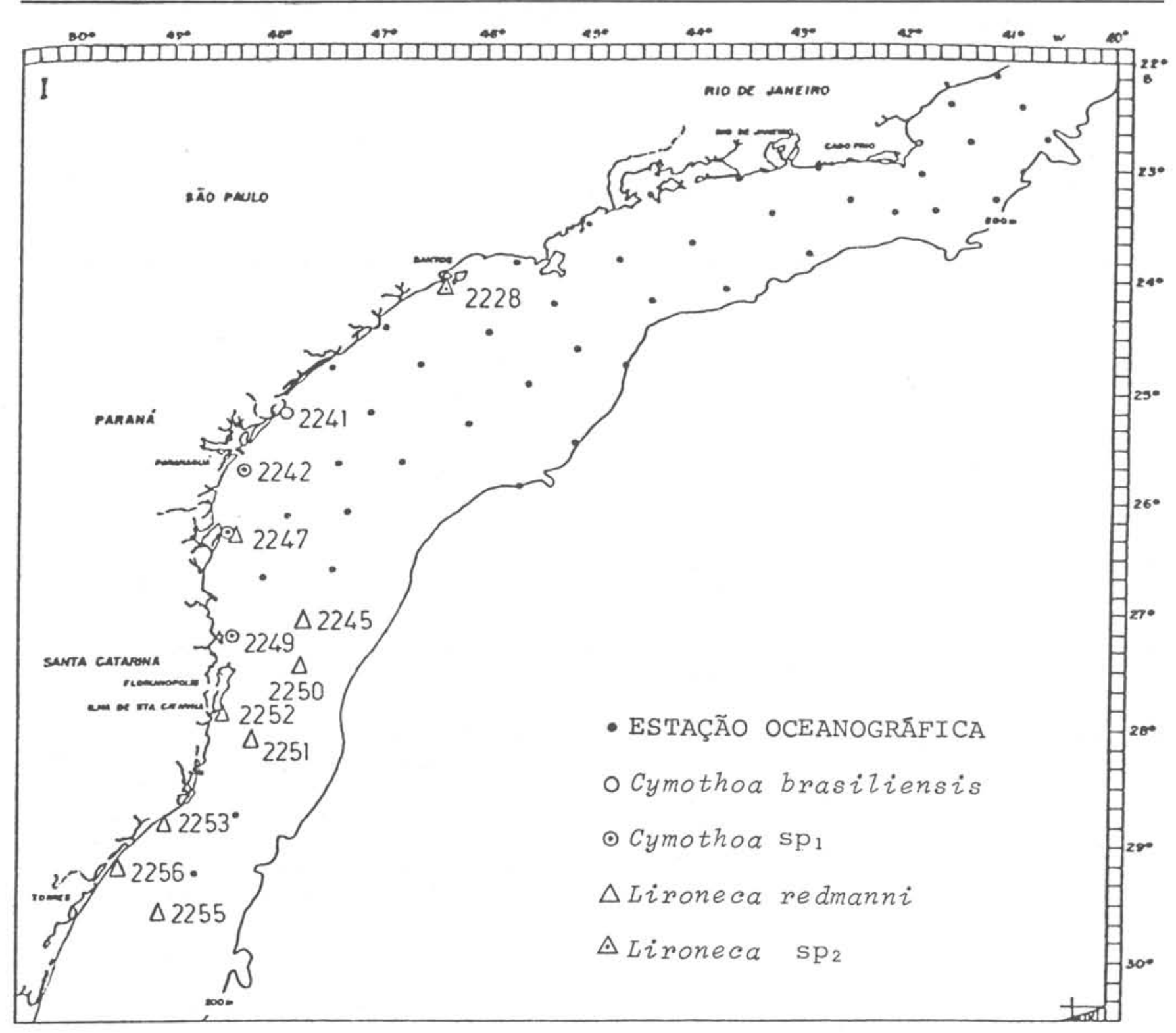

Fig. I. Localização das estações oceanogräficas realizadas durante o verão, com ocorrência de peixes parasitados por isöpodes cimotoídeos nas estações numeradas.

estações estiveram distribuídas entre profundidades de 10 e $200 \mathrm{~m}$. A presença de isópodes foi verificada examinando-se a região externa do corpo, boca e brânquias de cada peixe.

Em cada estação foram obtidos dados sobre temperatura, salinidade e teor de oxigênio dissolvido na camada de água próxima ao fundo. Esses valores, para as estaçôes de ocorrência de peixes parasitados encontram-se relacionados na Tabela 1. As variações observadas para os parâmetros supra citados nas estações de coletas e naquelas de ocorrência de parasitismo encontram-se na Tabela 2. o comprimento de cada isópode foi tomado em vista dorsal, da margem ântero-mediana da cabeça ao ápice do pleostelso, excluindo os urópodes, sob microscópio estereoscópico e com auxîlio de câmara clara.

\section{Espécies parasitas encontradas}

Foram examinados 90.418 exemplares de peixes, pertencentes a 186 espécies listadas no trabalho de Vazzoler et al. (1982). Na Tabela 3 é dado o índice de parasitismo observado nas espécies de peixes susceptíveis aos isópodes. Todos os isópodes parasitas encontrados pertencem à família Cymothoidae, 
Tabela 1. Posição das estações oceanogräficas com ocorrência de peixes parasitados por isópodes, profundidade, temperatura, salinidade $\epsilon$ teor de oxigênio dissolvido na camada de água pröximo ao fundo, durante as quatro estações do ano

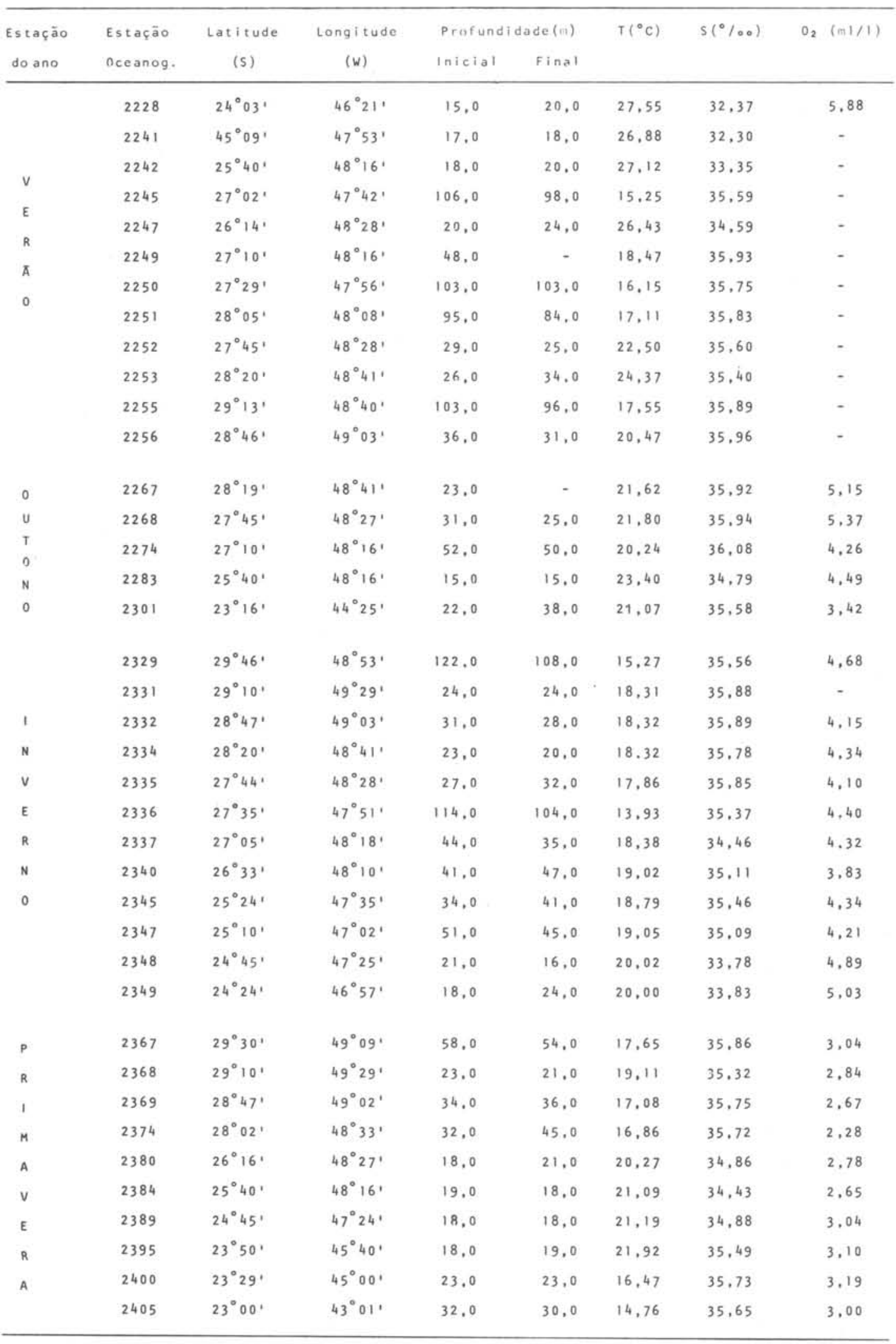


Tabela 2. Oscilação das variäveis: profundidade, salinidade, temperatura e oxigênio dissolvido na camada de ägua pröxima ao fundo, por estação do ano, para toda a região estudada $(T)$ e para os locais com ocorrência de parasitismo por isópodes (P)

\begin{tabular}{|c|c|c|c|c|c|c|c|c|c|c|c|c|c|c|c|c|}
\hline \multirow{2}{*}{ Estação do ano } & \multicolumn{4}{|c|}{ Profundidade (m) } & \multicolumn{4}{|c|}{ Salinidade $(\%)$} & \multicolumn{4}{|c|}{ Temperatura $\left({ }^{\circ} \mathrm{c}\right)$} & \multicolumn{4}{|c|}{ Oxigénio Dissolvido $(m 1 / 1)$} \\
\hline & & $T$ & & p & & $T$ & & $p$ & & $T$ & & P & & 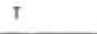 & & $p$ \\
\hline Verâo & 13 & -220 & is & -106 & 32.30 & $-36,79$ & 32,37 & -35.96 & 13.43 & -27.55 & 15.25 & -27.55 & 4.87 & $-7,22$ & $\cdot$ & $\cdot$ \\
\hline Outono & 13 & -210 & 15 & -52 & 34,36 & $-36,13$ & 34,79 & -35.92 & 14.48 & -23.76 & 20,24 & -23.40 & 2.94 & -5.34 & 4,26 & -5.37 \\
\hline Inverno & 10 & -122 & 20 & -122 & 33,56 & $=36,11$ & 33.78 & $-35,89$ & 13.90 & $-20,40$ & 15.27 & $-20,02$ & 3.79 & -5.20 & 3.83 & -4.89 \\
\hline Príanera & 15 & -132 & 18 & $=58$ & 34,15 & $-35,92$ & 34,43 & -35.75 & 13.50 & $-22,34$ & 14,76 & $-21,92$ & 2,03 & $-3,11$ & 2.65 & - \\
\hline
\end{tabular}

num total de 5 gêneros e 12 espécies, a seguir relacionados.

\section{Cymothoa $\mathrm{sp}_{1}$}

Apresentou o maior indice de ocorrência, num total de 711 exemplares parasitando 287 peixes da espécie

chloroscombrus chrysurus. As estações

de ocorrência estão assinaladas nas

Figuras 1-4.

Relação dos exemplares: 92 fêmeas pré-ovígeras $(13,00-27,50 \mathrm{~mm}), 122 \mathrm{f} \hat{\mathrm{e}}-$ meas ovígeras $(13,00-27,50 \mathrm{~mm}), 31 \mathrm{f} \hat{\mathrm{e}}-$ meas pós-ovígeras $(13,00-27,50 \mathrm{~mm})$, 236 machos adultos $(5,50-14,00 \mathrm{~mm})$, 230 jovens $(0,70-2,90 \mathrm{~mm})$.

Nota: 0 número de parasitas varia de 1 a 8 em cada hospedeiro, sendo 2 ou 3 o número mais freqüente. Quando o hospedeiro possui 2 parasitas, normalmente, um destes é macho e outro é fêmea. Quando o número é superior a dois, o restante é constituído pelos estädios juvenis. A fêmea localiza-se sempre na cavidade bucal, sobre a língua e o macho adulto ocupa a câmara branquial direita ou esquerda, indiferentemente (Fig. 5). Ambos apresentam a cabeça dirigida para a extremidade anterior do hospedeiro. Os individuos em estádios juvenís têm localização variável, ocorrendo na boca, ou mais comumente, nas brânquias, notadamente sobre os rastros dos arcos branquiais. Um estudo sobre o desenvolvimento, ciclo de vida, reprodução e relações com o hospedeiro pode ser encontrado em Sartor (1981).

Cymothoa brasiliensis schioedte \& Meinert, 1884

Relação dos exemplares: 1 fềmea pré-oví- gera $(12,00 \mathrm{~mm})$; 1 fêmea ovígera $(11,50 \mathrm{~mm})$; 1 fêmea pós-ovígera $(15,00 \mathrm{~mm}$ ) (Estações 2241, $2301 \mathrm{e}$ 2369 respectivamente, Figs 1, 2 e 4).

Nota: Pelo processo de conservação e descongelamento os parasitas se deslocaram antes que o hospedeiro fosse identificado.

Cymothoa excisa Perty, 1830

Relação dos exemplares: 1 fêmea ovígera $(23,50 \mathrm{~mm})$, na boca e 1 macho $(10,00 \mathrm{~mm})$, na câmara branquial de Orthopristes ruber (Cuvier, 1830); estação 2400, Fig. 4; 1 fêmea prē-ovígera $(25,00 \mathrm{~mm})$, jä destacada do hospedeiro; estação 2335, Fig. 3 .

\section{Cymothoa $\mathrm{sp}_{2}$}

Apenas um exemplar macho de 9,00 mm foi coletado, parasitando a cavidade bucal de Netuma barba (Lacépède, 1803); estação 2337 , latitude $27^{\circ} 05^{\prime}$ S, Fig. 3 .

\section{Lironeca redmanni Leach, 1818}

Ocorreu em grande número, num total de 125 exemplares, parasitando 125 peixes pertencentes a 7 espécies. As estações de ocorrência encontram-se assinaladas nas Figs 1-4.

Relação dos exemplares: 46 fêmeas pré-ovígeras, 32 fêmeas ovígeras, 4 fêmeas pós-ovígeras $(15,00-33,00 \mathrm{~mm}) ; 8$, em transição sexual $(15,00-18,50 \mathrm{~mm})$ e 12 machos $(12,50-16,50 \mathrm{~mm})$ em Cynoscion striatus (Cuvier, 1829); estações de ocorrência assinaladas nas Figs 1-4; 1 fêmea pré-ovígera, 2 fêmeas ovígeras, 3 fêmeas pós-ovígeras $(16,00-19,00 \mathrm{~mm})$ e 1 macho $(11,10 \mathrm{~mm})$ em Cynoscion jamai- 


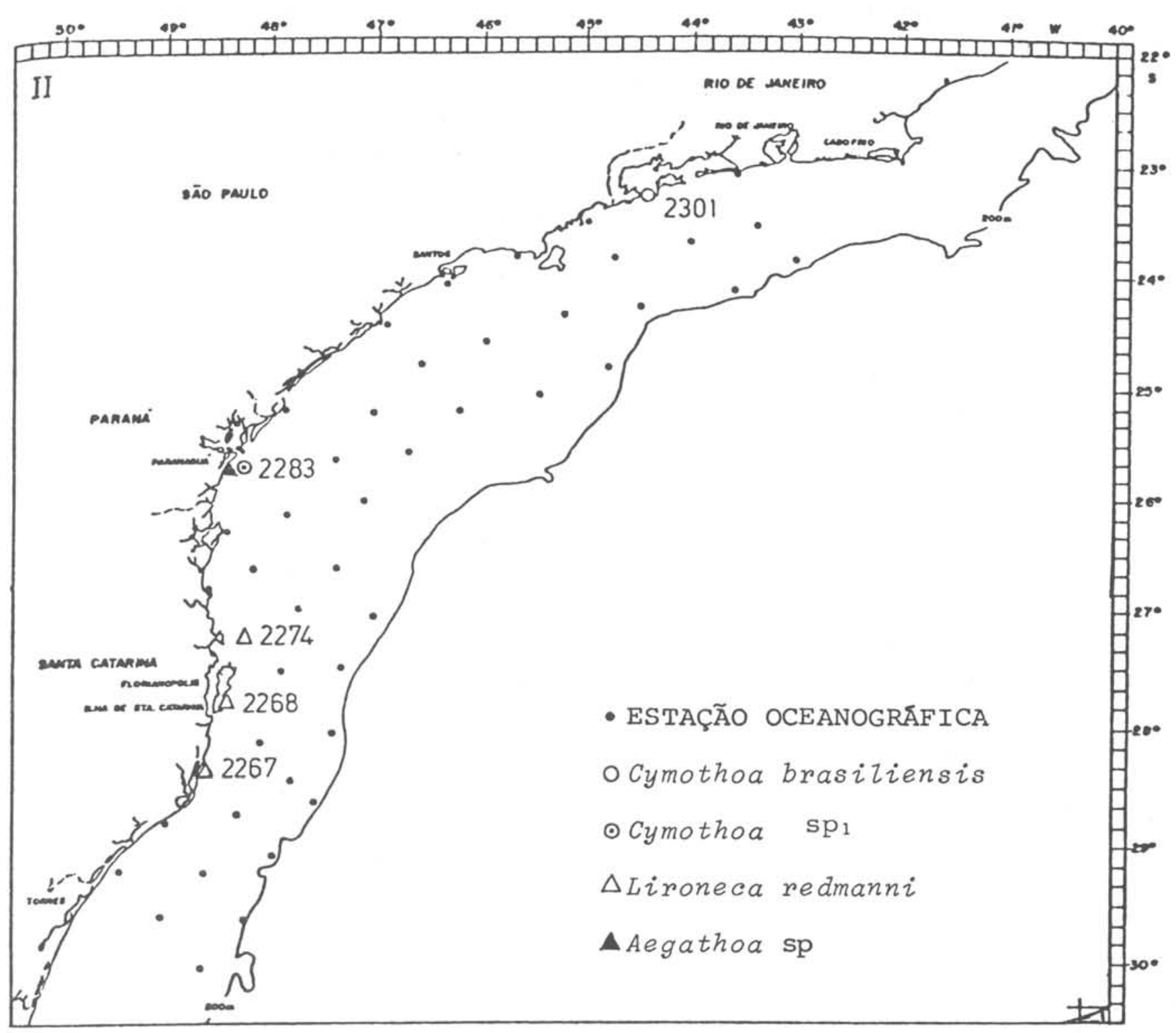

Fig. 2. Localização das estações oceanogräficas realizadas durante o outono, com ocorrência de peixes parasitados por isópodes cimotoídeos nas estações numeradas.

censis (Vaillant \& Bocourt, 1883); estações 2250, 2252, 2274 e 2334, Figs 1-3; 1 fêmea pré-ovígera, 2 fêmeas ovígeras $(16,00-19,00 \mathrm{~mm})$ em Ctenosciaena gracilicirrhus (Metzelaar, 1919); estações 2335, 2337 e 2340, Fig. 3; 1 fêmea pré-ovígera, 2 fêmeas ovígeras $(17,50$ $20,00 \mathrm{~mm}$ ) em Micropogonias furnieri (Desmarest, 1823); estações 2332 e 2337, Fig. 3; 1 fêmea pré-ovígera, 1 fêmea ovígera e 1 fêmea pós-ovígera (17,00 $19,00 \mathrm{~mm})$, em Orthopristis ruber (Cuvier, 1830); estações 2332 e 2400, Figs 3-4; 1 fêmea ovígera $(24,00 \mathrm{~mm})$ em Thyrsitops lepidopoides (Cuvier, 1829); estação 2250, Fig. 1; 2 fêmeas prē-oví- geras, 1 fêmea ovígera $(17,00-22,50$ $\mathrm{mm}), 1$ em transição sexual $(17,00 \mathrm{~mm})$ e 2 machos (15,50 - 16,00 mm) em Umbrina canosai Berg, 1895; estações 2332, 2335 e 2369, Figs 3 e 4.

Notas: Em cada um dos hospedeiros foi encontrado apenas um exemplar de $L$. redmanni, macho, fêmea ou em transição sexual, localizado sempre na câmara branquial direita ou esquerda, com a cabeça dirigida para a região anterior do peixe. A maior parte dos parasitas desta espécie ocorreu em exemplares de Cynoscion striatus. Alguns aspectos de sua relação com este hospedeiro são relatados em Sartor (1981). 


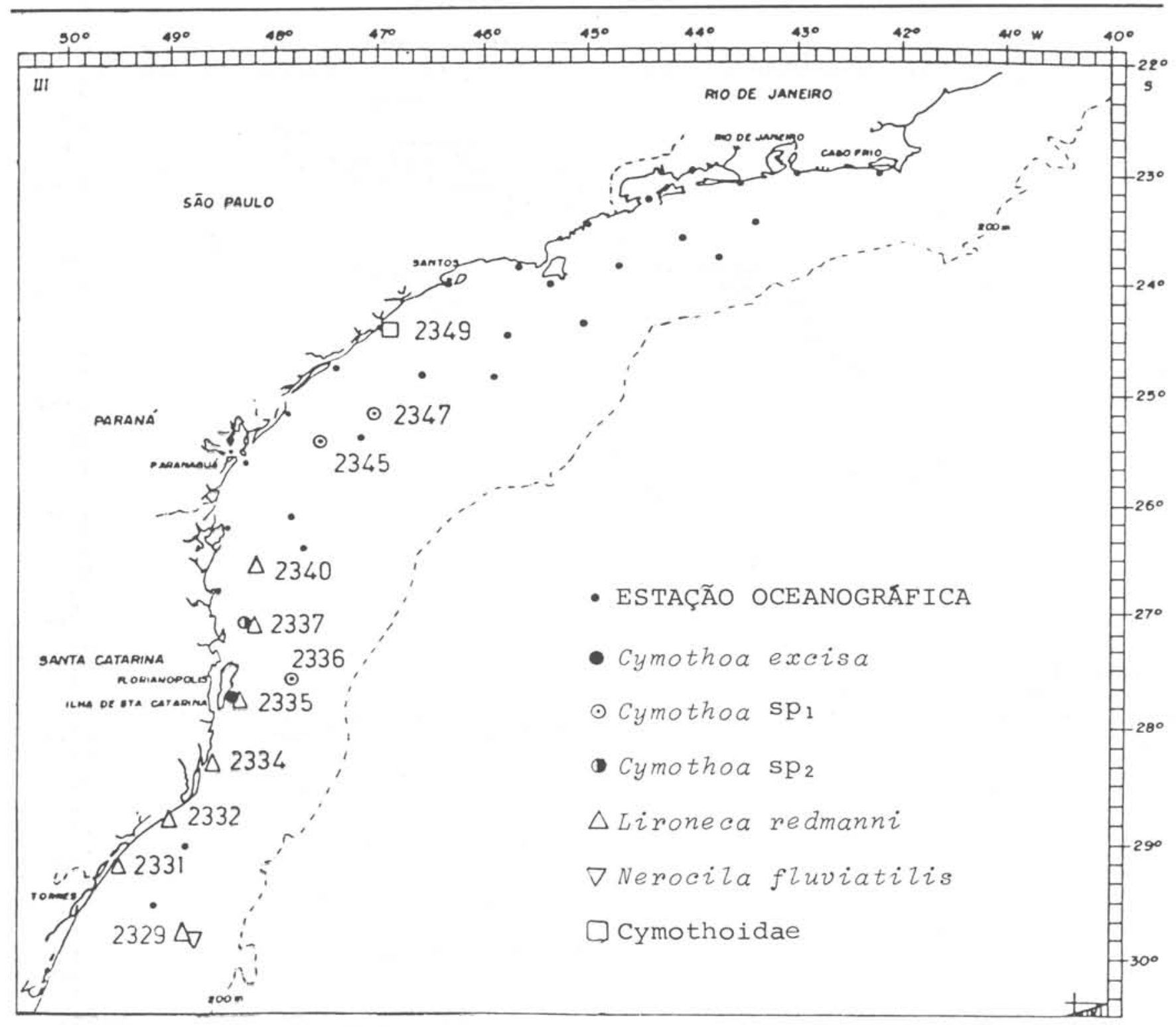

Fig. 3. Localização das estações oceanogräficas realizadas durante o inverno, com ocorrência de peixes parasitados por isópodes cimotoídeos nas estações numeradas.

Lironeca.sp 1

Relação dos exemplares: 1 fêmea pré-ovígera $(22,00 \mathrm{~mm})$ e um jovem $(5,00 \mathrm{~mm})$, ambos em um exemplar de Squalus cubensis Howe11-Rivero, 1936; estação 2405, 1atitude $23^{\circ} 00^{\prime} 00^{\prime}$ 's, Fig. 4.

\section{Lironeca $\mathrm{sp}_{2}$}

Relação dos exemplares: 1 fêmea ovígera $(18,5 \mathrm{~mm})$ em Cetengraulis edentulus (Cuvier, 1828); estação 2228, latitude $24^{\circ} 03^{\prime} 00^{\prime}$ 'S, Fig. 4 .

Nota: Durante todo o projeto, apenas um exemplar de $C$. edentulus foi coletado e, este único exemplar, se encontrava parasitado.

\section{Lironeca $\mathrm{sp}_{3}$}

Relação dos exemplares: 1 fêmea ovígera $(15,0 \mathrm{~mm})$ em Eucinostomus argenteus (Baird \& Girard, 1858); estaçāo 2380, latitude, $26^{\circ} 16^{\prime} 30^{\prime \prime} \mathrm{s}$, Fig. 4.

Nerocila armata Dana, 1852

Relação dos exemplares: 1 macho (24,00 mm); estaçăo 2384, latitude $25^{\circ} 40^{\prime} 30^{\prime \prime} \mathrm{S}, \mathrm{Fig} .4$

Nota: 0 parasita provavelmente ocorre na região externa da pele, sobre o peixe, e destacou-se devido ao processo de congelamento e descongelamento do material, impedindo a identificação do hospedeiro. 


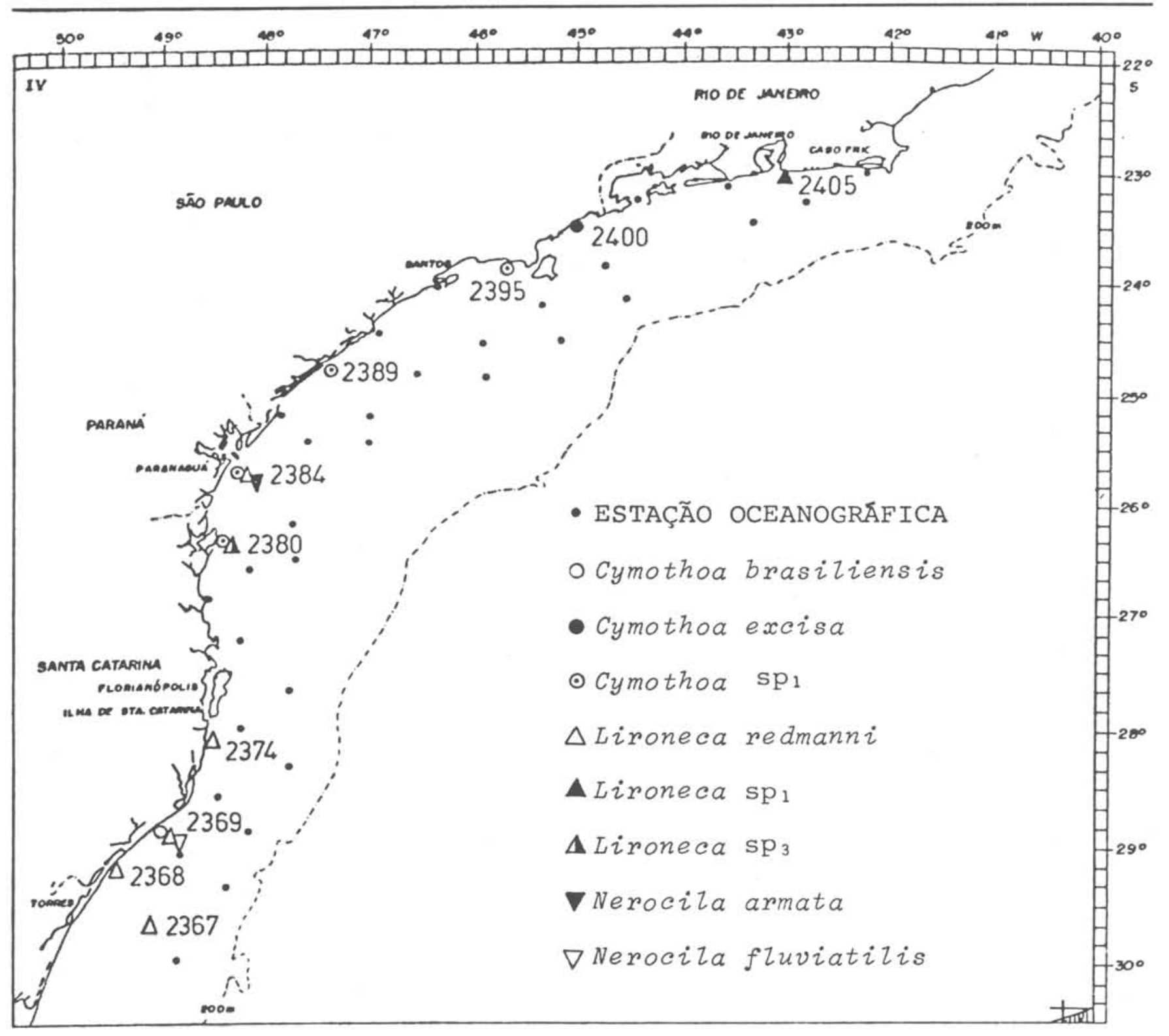

Fig. 4. Localização das estações oceanogräficas realizadas durante a primavera, com ocorrência de peixes parasitados por isöpodes cimotoídeos nas estações numeradas.

Nerocila fluviatilis Schiodte \& Meinert, 1884

Relação dos exemplares: 2 fêmeas pré-ovígeras $(27,00-28,50 \mathrm{~mm}), 1$ macho $(21,00 \mathrm{~mm})$, estações 2329 e 2369 , latitudes $28^{\circ} 47^{\prime} 12^{\prime \prime}$ 's e $29^{\circ} 48^{\prime} 00^{\prime}$ 's, Figs 3-4.

Nota: O parasita provavelmente ocorre na região externa da pele, sobre o peixe, e destacou-se devido ao processo de congelamento e descongelamento do material, impedindo a identificação do hospedeiro.

Aegathoa sp.

Relação dos exemplares: 1 macho $(8,50 \mathrm{~mm})$ em Chloroscombrus chrysurus;
(Linnaeus, 1766); estação 2283, 1atitude $25^{\circ} 40^{\prime} 00^{\prime}$ 's, Fig. 2.

Nota: 0 parasita ocorreu na cavidade bucal com sua cabeça voltada para a regiäo anterior do hospedeiro.

Gênero não determinado

Relação dos exemplares: 1 macho $(11,50 \mathrm{~mm}$ ) em Pellona harroweri (Fowler, 1917), estação 2349 , 1atitude

$24^{\circ} 24^{\prime} 00^{\prime}$ 's, Fig. 3.

Nota: Este exemplar pertence à família Cymothoidae mas não se enquadra dentro de nenhum dos gêneros conhecidos. Trata-se, provavelmente, de um novo gênero. 


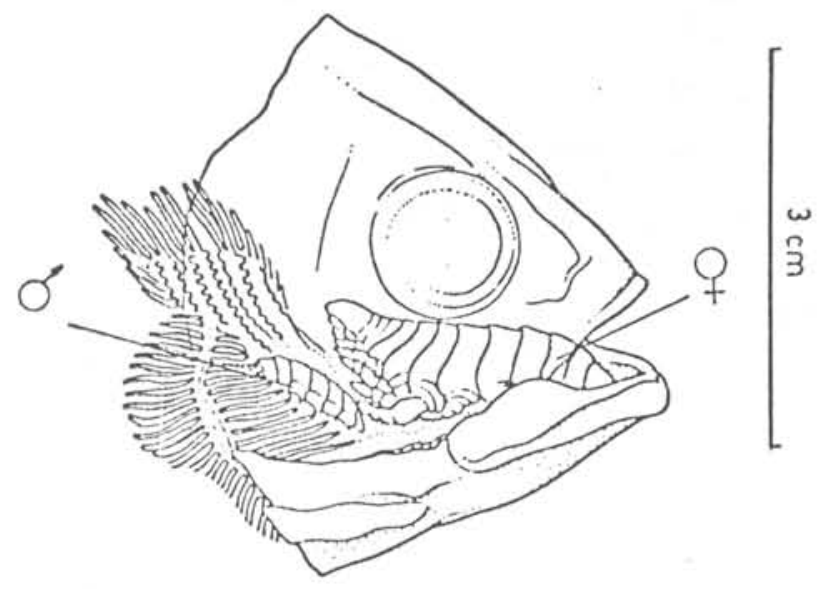

Fig. 5. Localização da fêmea e do macho de Cymothoa $\mathrm{sp}_{1}$ no hospede iro Chloroscombrus chrysurus.

\section{Discussão}

\section{Incidência de parasitismo}

Muitas das espécies de peixes examinadas foram coletadas em pequeno número e nelas não foram encontrados parasitas, o que não exclui entretanto, a possibilidade de vārias dessas espécies serem susceptíveis ao parasitismo por isópodes. Cordero (1937) e Moreira (1973), por exemplo, assinalaram Mustelus canis como sendo parasitado por isópodes na região do Rio da Prata - Uruguai e no Brasil, respectivamente. No presente estudo, entretanto, apenas sete exemplares desta espécie de peixe foram capturados sem que qualquer isópodes cimotoídeo fosse encontrado como parasita.

0 mesmo problema de amostragem nos impede de saber a real incidência do parasita Lironeca $\mathrm{sp}_{2}$ em Cetengraulis edentulus, jā que, como se observa pela Tabela 6, o unico exemplar do peixe coletado durante todo o período de estudo se apresentava parasitado. Na região de Cananéia (SP) muitos exemplares deste mesmo gênero de peixe são vítimas dos isópodes parasitas (Passos, 1978)*.

A porcentagem de peixes vítimas dos isópodes parasitas é muito variável e depende tanto da região estudada como da espécie de cimotoídeo considerada. Trilles (1962) encontrou até $80 \%$ de peixes da espécie Spicara moena parasitados por Emetha audouinii, enquanto

(*) Passos, M. J. de A. C. R. - São Paulo, Instituto Oceanogrä́fico, 1978. (Comunicação verbal).
Rouse (1969) relata mais de $90 \%$ de incidência de Cymothoa excisa em Orthopristis chrysopterus. Entretanto, baixos Índices de parasitismo são comumente encontrados (Trilles, 1964a,b; Willians, 1974; Capapé \& Pantoustier, 1976). No presente estudo, a maior parte dos hospedeiros apresentou baixa taxa de infestação (Tab. 3). A espécie de peixes Cynoscion striatus apresentou a incidência máxima de $22,22 \%$, enquanto Chloros combrus chrysusus chegou a apresentar $44,91 \%$ de indivíduos parasitados na região de Paranaguá (PR), durante o verão. Esta região demonstrou ser um local bastante favorável à ocorrência de Cymothoa $\mathrm{sp}_{1}$ em $C$. chrysurus. A oscilação nas variáveis físico-químicas, consideradas neste estudo, não auxiliaram na explicação dessa aparente alta concentração, e nem se mostrou relacionada com a ocorrência das demais espécies parasitas.

\section{Especificidade parasitária}

Apesar da maioria das espécies parasitas assinaladas na área terem sido coletadas em pequeno número, dificultando uma análise referente $\bar{a}$ especificidade, nota-se que apenas para Lironeca redmanni foi observada mais de uma espécie hospedeira.

0 parasita Cymothoa $\mathrm{sp}_{1}$ coletado em grande número, mostrou ser altamente específico ao hospedeiro Chloroscombrus chrysurus, não ocorrendo em nenhuma outra espécie de peixe. Lironeca redmanni ocorreu em sete espécies diferentes pertencentes a três famílias, principalmente em Sciaenidae, mostrando certa preferência pela espécie Cynoscion striatus.

värios pesquisadores têm observado alto grau de especificidade para algumas éspécies de isópodes parasitas (Menzies, Bowman \& Alverson, 1955; Bowman, 1960; Fryer, 1968; Kroger \& Guthrie, 1972; Linco1n, 1972; Morton, 1974; Taberner, 1979), enquanto outros não encontraram preferência dos isópodes por uma espécie particular de peixe (Comeaux, 1942; Legrand, 1952; Trilles, 1962; Cheng, 1964; Roman, 1970; Brusca 1978a,b). Como Trilles (1964a) mostrou, o grau de especificidade parasitāria é variável, dependendo do parasita considerado, não sendo possível uma generalização para os cimotoídeos.

Roman (1970) colocou em dúvida a especificidade assinalada na literatura, considerando que isto poderia ser devido 
Tabela 3. Relação das famílias e espécies de peixes que apresentaram parasitismo por isópodes; número coletado, nümero parasitado e índice de parasitismo por estação do ano de 1975

\begin{tabular}{|c|c|c|c|c|c|c|c|c|c|c|c|c|c|}
\hline \multirow{3}{*}{ Famflile } & \multirow{3}{*}{ Espécie } & \multicolumn{3}{|c|}{ Veräo } & \multirow{2}{*}{\multicolumn{2}{|c|}{$\begin{array}{c}\text { Outono } \\
\text { N? }\end{array}$}} & \multicolumn{4}{|c|}{ Inverno } & \multicolumn{3}{|c|}{ Primavera } \\
\hline & & N? & N: & & & & & Ne & N: & & N\% & Ne & \\
\hline & & - 1. & Par. & $z$ & col. & Par. & $z$ & col. & Par. & 8 & col. & par. & 2 \\
\hline Aritioas & Netuma barba (Lacápède, 1803) & 9 & 0 & 0 & 473 & 0 & 。 & 223 & 1 & 0,45 & 79 & 0 & 。 \\
\hline Carangidae & Cheonoscombrus chrysurus (Linnaeus, 1766) & 1177 & 80 & 6,80 & 236 & 62 & 26.27 & 131 & 4 & 2,48 & 502 & 141 & 28,09 \\
\hline Clupeidas & Peltona hantoweni (Fowler, 1917) & 128 & 0 & 0 & 163 & 0 & 。 & 1054 & 0 & 0 & 215 & 1 & 0,47 \\
\hline Engraullidae & Cetengraulis edentulus (Cuvier, 1829) & 1 & 1 & - & 0 & 0 & 0 & 0 & 0 & 。 & 0 & 0 & 0 \\
\hline Gempyilidae & Thynsitops lepidopoides (Cuvier, 1829) & 322 & 1 & 0.31 & 112 & o & 0 & 26 & 0 & 0 & 736 & 0 & 0 \\
\hline Gerreldae & Eucinostomus angenteus (Baird. Givard, 1854) & 42 & 0 & 0 & 5 & 0 & o & 15 & 0 & 0 & 57 & 1 & 1,75 \\
\hline Pomadesyleae & Orchepristis suber (Cuvier, 1830) & 1 & 0 & 0 & 179 & - & ० & 141 & 2 & 1,42 & 35 & 1 & 2,86 \\
\hline \multirow[t]{5}{*}{ Sclaentidae } & Ctenosciaena gracilicirshus (Metzelas, 1919) & 1481 & 0 & 0 & 1607 & o & 。 & 3500 & 4 & 0,11 & 5407 & a & 0 \\
\hline & cynoscion famaicensis (veillant s Bocourt, 1883) & 766 & 2 & 0,26 & 250 & 1 & 0,40 & 651 & 4 & 0.61 & 637 & o & 。 \\
\hline & Cunoscien striatus (Cuvier, 1829) & 812 & 10 & 1,23 & 449 & 10 & 2.23 & 2464 & 73 & 2.96 & 276 & 8 & 2.90 \\
\hline & Mieropogoniags furnieri (Desmarest, 1823). & 125 & 0 & 0 & 226 & 0 & 0 & 522 & 2 & 0,38 & 351 & 0 & o \\
\hline & Umbnina canosai (Berg. 1895) & 116 & 。 & 0 & 157 & 0 & 0 & 1291 & 6 & 0.46 & 1814 & 6 & 0,38 \\
\hline Squalidee & Squalus cubensis (Howel1-Rivero, 1936) & 3 & 0 & 0 & 26 & o & . & 22 & 0 & 0 & 16 & 1 & 6,25 \\
\hline
\end{tabular}

à uma abundância de certas espécies de peixes em relação à outras. Entretanto, parece não ser este o motivo para a especificidade observada em Cymothoa $\mathrm{sp}_{1}$. Outros peixes da família Carangidae também foram coletados em grande número nas mesmas estaçöes de ocorrência de C. chrysurus, sem que portassem qualquer isópode. A grande ocorrência de vários peixes susceptiveis ao parasitismo por outras espécies de cimotoídeos, nas mesmas estações de coleta de $C$. chrysurus parasitados por Cymothoa $\mathrm{sp}_{1}, 1 \mathrm{e}^{-}$ vam-nos a discordar das idéias de Roman (op. cit.). Os dados aqui apresentados indicam haver especificidade entre parasita e hospedeiro, pelo menos para Cymothoa spi e C. chrysurus.

\section{Número e localização do parasita no hospedeiro}

0 número de exemplares de Lironeca redmanni encontrados em um mesmo hospedeiro foi constante e igual a 1 , enquanto que Cymothoa spi ocorreu em número variável de 1 a 8 . Em alguns casos certa constância é observada (Trilles 1964b;

Turner \& Roe, 1967; Bowman \& Mariscal, 1968) mas, mais freqüentemente, o número de isópodes presentes è variável, mesmo quando se considera uma espécie particular de parasita (Castro \& Machado Filho, 1946; Roman, 1970; Willians \& Willians, 1978).

Parece ser rara a ocorrência de um número elevado de isópodes num mesmo peixe, como foi observado para Cymothoa $\mathrm{sp}_{1}$. Mais que 3-4 parasitas por hospedeiro è relatado apenas por Inouye (1941) e Trilles (1962).

Os cimotoídeos podem se localizar em diferentes regiões do corpo do hospedeiro, existindo, porém, constância no 1ocal escolhido para cada espécie. A maioria das espécies do gênero Cymothoa ocorrem na boca do hospedeiro (Lanchester, 1902; Trilles, 1975a, Galzin \& Trilles, 1979), algumas, porém, podem ser encontradas na câmara branquial do peixe (Trilles, 1975b). Cymothoa spi tem localização muito característica, com a fêmea na boca e o macho em uma das câmaras branquiais. Ambos apresentam a cabeça voltada para a porção anterior do hospedeiro, tal como o observado para várias outras espécies (Castro \& Machado Filho, 1946; Bowman, 1960; Trilles, 1964b). Menzies et al. (1955) e Taberner (1979), entretanto, encontraram a fêmea de Lironeca convexa e Isonebula maculata respectivamente, dirigidas para a região caudal do peixe.

Lagarrique \& Trilles (1969) incluem 。 gênero Lironeca entre os parasitas considerados "branquiais". Esta localização parece realmente ser a mais comum para o gênero, apesar de algumas espēcies, como L. expansus, ocorrer na boca (Fryer, 1965). Lironeca redmanni, concordante com o padrão geral, ocorre na câmara branquial e, também, orientada para a região anterior do hospedeiro. 
Bolm Inst. oceanogr., S Paulo, 34(ünico), 1986

\section{Resumo}

No presente trabalho examinaram-se 90.418 exemplares de 186 espécies de peixes, coletados entre a região de Cabo de São Tomé (RJ) e Torres (RS) - Brasil (1atitudes $22^{\circ} 27^{\prime} 00^{\prime}$ 's $-29^{\circ} 21^{\prime} 00^{\prime}$ 's), o que possibilitou um levantamento das espécies de isópodes parasitas que ocorrem na área e das espécies de peixes susceptíveis a este parasitismo. Foram constatadas 12 diferentes espécies de isópodes da família Cymothoidae e 13 espécies hospedeiras.

o Indice de parasitismo observado foi variável dependendo das espécies de parasita e hospedeiro consideradas, local de coleta e época do ano. 0 peixe Chloroscombrus chrysurus apresentou o maior indice de parasitismo entre as espécies analisadas, com ārea de concentração na região prōxima a Paranaguá (PR), (latitude $25^{\circ} 40^{\prime} 00^{\prime}$ 's).

Foi encontrado alto grau de especificidade de Cymothoa spi com relação ao hospedeiro $C$. chrysurus. Lironeca redmanni entretanto, apesar de parasitar principalmente peixes da espécie Cynoscion striatus parece não ser parasita específico do mesmo, pois ocorreu também em outras seis espécies hospedeiras.

\section{Agradecimentos}

Agradeço ao Prof. Dr Alceu Lemos de Castro pelo auxílio na identificação das espécies de isópodes; ao Dr Gelso Vazzoler, Chefe-científico do Projeto FAUNEC; à Dra Ana Maria Setubal Pires Vanin pelo apoio e revisão do manuscrito, ao Conselho Nacional de Desenvolvimento Científico e Tecnológico (CNPq) pela bolsa de aperfeiçoamento concedida durante a realizaçao deste trabalho.

\section{Referências bibliográficas}

BOWMAN, T. E. 1960. Description and notes on the biology of Lironeca puhi, n.sp (Isopoda Cymothoidae), parasite of the Hawaiian moray eel, Gymnothorax eurostus (Abbott). Crustaceana, 1(2): 84-91.

\& MARISCAL, R. N. 1968. Renocila heterozota a new cymothoid isopod, with notes on its host, the anemone fish, Amphiprion akallopisos, in the Seychelles. Crustaceana, 14(1): 97-104.
BRUSCA, R. C. 1978a. Studies on the cymothoid fish symbionts of the eastern Pacific (Crustacea: Isopoda: Cymothoidae). I. Biology of Nerocila californica. Crustaceana, 34 (2): 141-154.

1978b. Studies on the cymothoid fish symbionts of the eastern Pacific (Crustacea: Isopoda: Cymothoidae). II. Systematics and biology of Lironeca vulgaris Stimpson 1857. Occ. Pap. Allan Hancock Fdn, n.s., (2):1-19.

CAPAPE, C. \& PANTOUSTIER, G. 1976. Liste commentée des isopodes parasites de sélaciens des côtes tunisiennes. I. Côtes septentrionales, de Tabarka à Bizerte. Archs Inst. Pasteur Tunis, 3:197-210.

CARVAlHo, J. P. 1939. Sobre um caso curioso de ectoparasitismo. Revta Ind. anim., n.s., 2(3):41-44.

CASTRO, A. L. 1955. Paracymothoa astyanaxi g. n. e sp n. de isópode parasita de peixe de água doce (Isopoda, Cymothoidae). Revta bras. Biol., 15(4):411-414.

\section{\& MACHADO FILHO, J. P.} 1946. Artystone trysibia Schioedte, um crustáceo parasita de peixe dágua doce do Brasil, com descrição do alótipo macho (Isopoda, Cymothoidae). Revta bras. Biol., 6(3):407413.

CHENG, T. C. 1964. The biology of animal parasites. Philadelphia, W. B. Saunders, 727 p.

COMEAUX, G. T. 1942. Parasitic isopods of fishes from the Grand Isle, Louisiana region. (Abstract). Proc. LA Acad. Sci., 6:86.

CORDERO, E. H. 1937. Nerocila fluviatilis y otros isópodos parásitos de las familias Cymothoidae y Bopyridae del Uruguay y del Brasil. An. Mus. Hist. nat. Montevideo, ser. 2, 4(12):1-11. 
DANA, J. D. 1852. Crustacea. United States Exploring Expedition, during the years $1838 / 42$, under the command of Charles Wilkes, U.S.N. vol. 14 part 2, p. 691-805.

FRYER, G. 1965. A new isopod of the genus Lironeca, parasitic on a cichlid fish of Lake Tanganyika. Revue Zoo1. Bot. afr., 71:376-384.

1968. A new parasitic isopod of the family Cymothoidae from clupeid fishes of Lake Tanganyika - a further Lake Tanganyika enigma. J. Zool., Lond., 156:35-43.

GALZIN, R. \& TRILLES, J. P. 1979. Sur la présence de Cymothoa pulchrum Lanchester, 1902 (Isopoda, Flabellifera, Cymothoidae) en Polynésie Française. Crustaceana, $36(3): 257-266$.

INOUYE, M. 1941. On sexuality in Cymothoidae, Isopoda. 2. Irona melanosticta Schioedte and Meinert parasitic in the branchial cavity of the halfbeak. Hyphorhampus sajari (Temminck and Schlegel). J. Sci. Hiroshima Univ., sér. B, 9:219-238.

KROGER, K. L. \& GUTHRIE, J. F. 1972. Incidence of the parasitic isopod, olencira praegustator, in juvenile Atlantic menhaden. Copeia, (2) : 370-374.

LAGARRIQUE, J. G. \& TRILLES, J. P. 1969. Nouvelles recherches écologiques sur les isopodes Cymothoidae méditerranéens: I. L'importance; 1a calcification et les constituants organiques de la cuticule ses variations suivant les espēces. Vie Milieu, 20(1-A):117-136.

LANCHESTER, W. F. 1902. On the Crustacea collected during the "Skeat Expedition" to the Malay Peninsula. Proc. zool. Soc. Lond., 2:363-379.

LEGRAND, J. J. 1952. Contributions à l'étude expérimentale et statistique de 1a biologie d'Anilocra physodes L. (Crustacé Isopode Cymothoidé). Archs Zool. exp. gén., 89(1):1-56.
LINCOLN, R. J. 1972. A new species of Lironeca (Isopoda: Cymothoidae) parasitic on cichlid fishes in Lake Tanganyika. Bull. Br. Mus. nat. Hist. (Zool.), $21(8): 329-338$.

MENZIES, R. J.; BOWMAN, T. E. \& ALVERSON, F. G. 1955. Studies of the biology of the fish parasite Lironeca convexa Richardson (Crustacea, Is opoda, Cymothoidae). Wasmann J. Biol., 13(2):277-295.

MOREIRA, P. S. 1972. Species of marine Isopoda (Crustacea, Peracarida) from shouthern Brazil. Bolm Inst. oceanogr., S Paulo, 21:163-179.

\section{Espécies de} Isopoda (Crustacea, Peracarida). PRGS-II. Publção esp. Inst. oceanogr. Univ. S Paulo, (3, pt. 1): 213-229.

1977. Occurrence and ecological notes on Rocinela signata (Isopoda, Flabellifera) off Brazil. Bolm Inst. oceanogr., S Paulo, 26: 293-301.

\& SADOWSKY, V. 1978.

An annotated bibliography of parasitic Isopoda (Crustacea) of Chondrichthyes. Bolm Inst. oce anogr., S Paulo, 27(2):95-152.

MORTON, B. 1974. Host specificity and position on the host in Nerocila phaeopleura Bleeker (Isopoda, Cymothoidae). Crustaceana, 26(2): 143-148.

NOVOTNY, A. J. \& MAHNKEN, C. V. W. 1971. Predation on juvenile Pacific salmon by a marine isopod Rocinela belliceps pugettens is (Crustacea, Isopoda). Fishery Bu11., 69 (3): 699-701.

ROMAN, M. L. 1970. Contribution a 1'étude de la biologie des Cymothoidae (Crustacés Isopodes) de 1a Baie de la Ciotat. Tethys, 2(2):501-514.

ROUSE, L. W. 1969. Littoral Crustacea from southwest Florida. Q. J. Fla Acad. Sci., 32(2):127152 . 
SARTOR, S. M. 1981. Isópodes parasitas de peixes marinhos (Flabellifera, Cymothoidae), na plataforma continental brasileira, entre as latitudes de $22^{\circ} 27^{\prime} 00^{\prime \prime S}$ e $29^{\circ} 21^{\prime} 00^{\prime \prime S}$, incluindo a biologia de Cymothoa sp. Dissertação de mestrado. Universidade de São Paulo, Instituto Oceanográfico, 129p.

SCHIOEDTE, J. C. \& MEINERT, F. 1884. Symbolae ad monographiam Cymothoarum crustacearum. Isopodum familiae. Naturh. Tidsskr., 14:221455.

SCHULTZ, G. A. 1969. How to know the marine isopod crustaceans. Dubuque, Iowa, W. C. Brown, 359p.

SZIDAT, L. \& SCHUBART, 0. 1960. Neue und Seltene Parasitische Süsswasser-Asseln der Familie Cymothoidae aus dem Rio Mogi Guassu, Brasilien (Isopoda). Anais Acad. bras. Ciênc., $32(1): 107-124$.

TABERNER, R. 1979. Datos para el conocimiento biológico de Paracymothoa parva Taberner 1976 e Isonebula maculata Taberner 1977 (Crustacea, Isopoda, Cymothoidae). Parasitologia, 2(4): 77-86.

TRILLES, J. P. 1962. Remarques morphologiques et biologiques sur les "Isopodes Cymothoidae" parasites de poissons de l'étang de Thau. Naturalia monspel. (Zool.), (3): 101-124.

\section{4a. Spécificité} parasitaire chez les isopodes Cymothoidae mediterranéens. Note préliminaire. Vie Milieu, 15(1): 105-116.

1964b. Un nouveau cymothoadien, Meinertia capri n. sp (Isopoda), parasite de Capros aper Lacépède, 1803 (Téléostéens, Caproidae) en Méditerranée. Crustaceana, 7:188-198.

1972. Les Cymothoidae (Isupoda, Flabellifera) des côtes françaises (systématique, faunistique, écologie et répartion géographique). Bul1. Mus. Hist. nat. (Zoo1.), sēr. 3, 70(91): 1191-1230.

1975a. Les Cymothoidae (Isopoda, Flabellifera) des collections du Muséum National d'Histoire Naturalle de Paris. II. Les Anilocridae Schioedte et Meinert, 1881. Genres Anilocra Leach, 1818 et Nerocila Leach, 1818. Bu11. Mus. natn Hist. nat., Paris, 3e sér. (290), (Zool, 200):303-346.

1975b. Les Cymothoidae

(Isopoda, Flabellifera) des collections du Muséum National d'Histoire Naturelle de Paris. III. Les Cymothoinae Schioedte et Meinert, 1884. Genre Cymothoa Fabricius, 1787. Bu11. Mus. natn Hist. nat., sér. 3, 318(Zool. 225): 977-993.

TUÊ, V. T. 1963. Sur la présence de dents vomériennes et ptérygoidiennes chez Boops boops (L.) (Pisces, Sparidae), en rapport avec l'isopode phorétique intrabucal Meinertia. Vie Milieu, 14(2):225-235.

TURNER, W. R. \& ROE, R. B. 1967. Occurrence of the parasitic isopod Olencira praegustator in the yellowfin menhaden, Brevoortia smithi. Trans. Am. Fish. Soc., 96(3):357-359.

VAZZOLER, G.; ZANETI-PRADO, E. M.; KAWAKAMI, E. \& YAMAGUTI, N. 1982. Teleósteos marinhos coletados entre Cabo Frio $\left(23^{\circ} \mathrm{S}\right)$ e Torres (29 $\left.21^{\circ} \mathrm{S}\right)$ - Programa FAUNEC (1975). Dusenia, $13(3): 127-133$.

WILLIAMS Jr, E. H. 1974. Treatments employed for control of parasites of selected fishes stocked in mariculture experiments (1969-1972). Proc. Wld maricult. Soc., 5:291-295.

\& WILLIAMS, L. B. 1978. Cymothoid isopods of some marine fishes from the northern Gulf of México. NE Gulf Sci., $2(2): 122-124$. 